\title{
On hydromagnetic oscillations in a rotating cavity
}

\author{
By ROGER F. GANS \\ Division of Geological and Planetary Sciences, $\uparrow$ California Institute of Technology, \\ Pasadena, California 91109
}

(Received 1 February 1971 and in revised form $20 \mathrm{July} \mathrm{1971)}$

Time-dependent hydromagnetic phenomena in a rotating spherical cavity are investigated in the framework of an interior boundary-layer expansion. The interior problem is shown to contain waves whose frequencies are of order $\omega, A \omega$ and $A^{2} \omega$, where $\omega$ is the rotation rate of the cavity and $A^{2}=B^{2} / 4 \pi \rho \omega^{2} R^{2} \ll 1$ is the Alfvén number. $B$ is an imposed magnetic field, $\rho$ the fluid density and $R$ the radius of the cavity. The first type of wave is a modification of the hydrodynamic inertial wave, the second is a pseudo-geostrophic wave and is involved in spin-up, and the third is related to the MAC waves of Braginskiy (1967).

It is shown that the MAC waves must satisfy more than the usual normal boundary conditions and that reference must be made to the boundary-layer solution to resolve the ambiguity regarding which conditions are to be taken. For normal liquid metals of small magnetic Prandtl number the MAC waves must satisfy full magnetic boundary conditions; only the no-slip conditions may be deferred to the boundary layers.

The boundary-layer structure is investigated in detail to display the interactions between applied field, viscosity, electrical conductivity, frequency and latitude. The decay of the pseudo-geostrophic modes, essentially the spin-up problem, is discussed for a non-axisymmetric constraining field and non-zero container conductivity. Three regimes exist, depending on container conductivity.

\section{Introduction}

This paper is addressed to the problem of free hydromagnetic oscillations in a contained rotating electrically conducting fluid in the presence of an applied magnetic field. Three types of oscillations exist in the geophysically relevant case of small magnetic field. This paper establishes the order of magnitude of the frequencies of these three types of modes, and of their decay rates. To do this it is necessary to discuss the boundary-layer structure in detail and $\$ 3$ gives a full exposition of hydromagnetic boundary layers in a sphere, displaying the interaction between viscosity, conductivity, constraining field and frequency. Because of the latitude-dependence of the boundary layers the relative importance of viscosity and conductivity can change and the effect of the dominance of each is considered.

There is some overlap with the work of Braginskiy (1967) in which waves with

$\dagger$ Present address: Department of Mathematics, Massachusetts Institute of Technology, U.S.A. 
frequency of the order of the rotation frequency and waves of very much lower frequency are shown to be possible. However, the emphasis in the present work is on the boundary layers associated with these waves and the effect these have on the eigenfrequencies. In particular it will be shown that the slow waves, for parameters appropriate to the earth, have frequencies determined by the boundary layers rather than the non-dissipative eigenvalue problem.

A matter of some novelty associated with the boundary layers is that, for the slowest modes, the dissipation-free boundary conditions are determined by the nature of the boundary layers. The usual circumstance in boundary-layer problems is that the dissipation-free problem is required to satisfy only normal boundary conditions, all the tangential boundary conditions being reserved for the boundary-layer solution. It will be shown that this is not the case in the present instance. Half the tangential boundary conditions must be satisfied by the dissipation-free solution. The choice of which tangential boundary conditions are to be satisfied cannot be made without examining the boundary layers. This lends novelty to the analysis but also makes the dissipation-free problem considerably more difficult, setting it beyond the analytic range of this paper.

If $\nu, \eta, \rho, \omega$ are the kinematic viscosity, electrical conductivity, density and rotation rate, and $L$ and $B$ represent characteristic length and magnetic field, the appropriate non-dimensional parameters may be written

$$
A^{2}=B^{2} / 4 \pi \rho \omega^{2} L^{2} ; \quad E=v / \omega L^{2} ; \quad E_{m}=\left(4 \pi \eta \omega L^{2}\right)^{-1} .
$$

These are the Alfvén, Ekman and magnetic Ekman numbers and they measure the characteristic Lorentz force and viscous and magnetic diffusion. The free oscillations of the system are given in the non-dissipative limit $E, E_{m} \rightarrow 0$. To apply this limit to physical systems it is necessary that $E$ and $E_{m}$ both be smaller than $A^{2}$. Thus $A^{2}$ is not a totally arbitrary quantity. This must be kept in mind when examining the results of the analysis. In particular the meaning of the limit $A^{2} \rightarrow 0$ must be carefully assessed; it will be discussed below only in a dissipative context where $E$ and $E_{m}$ are small but non-zero.

Braginskiy (1967) has discussed the special case in which magnetic field is linearized with respect to an average azimuthal field and in which bouyancy forces are considered. The complications introduced by buoyancy forces would obscure the physics of the boundary-layer phenomena considered in the present work and so the buoyancy forces have been neglected in what follows. Malkus (1967) examined a system similar to that considered by Braginskiy (1967) and Gans (1971) has considered a system for which the magnetic field is linearized about a uniform (force-free) axial field. Neither Braginskiy nor Malkus proceed to the dissipative level and none of these analyses specifically uncovers what will be called below the 'pseudo-geostrophic' mode.

The pseudo-geostrophic mode is the mode which would reduce to the ordinary geostrophic mode in the limit $A^{2} \rightarrow 0$ (in such a way that $E / A^{2}$ and $E_{m} / A^{2}$ also vanish). It can produce visible effects if the linearizing field has a poloidal component which is not axisymmetric. The symmetry of the linearizing field has kept this mode from previous consideration.

In a formal non-dissipative ordering scheme the eigenfrequencies associated 
with this mode will be of the order of $A \omega$. The slow modes previously considered have eigenfrequencies of the order of $A^{2} \omega$.

In this paper the analysis will be specialized to a spherical container of conductivity $\eta^{\prime}$. The conductivity ratio $\eta^{\prime} / \eta$ will be denoted by $S$. The field around which the system is to be linearized will be supposed to be the gradient of a potential. W. V. R. Malkus (personal communication) points out that this rules out instabilities; however, it provides field lines which penetrate the boundary, and a way of considering asymmetric basic fields. Were it possible to uncover the exact eigenfrequencies of the system the omission of potential instabilities would be serious, outweighing the benefits. Since this is not possible it is felt that the strong interaction at the boundaries, which this choice allows, justifies the choice. In an earth-like setting, as will be shown below (\$3), a toroidal field of $300 \mathrm{G}$ will have negligible effect on the boundary layers compared to a poloidal field of $5 \mathrm{G}$.

The plan of the paper is as follows. In $\S 2$ the problem is formulated, and the nature of eigenfrequencies is established. In $\$ 3$ the boundary conditions and the boundary layers necessary to complete them are discussed. In $\$ \S 4$ and 5 the pseudo-geostrophic modes are discussed. Section 6 presents a brief discussion.

\section{Formulation}

The dimensionless equations governing the system are those of the usual hydromagnetic approximation. Using the cavity radius as a length scale, the peripheral velocity as a velocity scale, the inverse rotation rate as a time scale and the magnitude of the basic field as a magnetic field scale one can define the non-dimensional numbers given by equation (1.1). In terms of these the basic equations are

$$
\left.\begin{array}{rl}
(\partial \mathbf{v} / \partial t)+2 \mathbf{k} \times \mathbf{v}+\nabla \Pi & =E \nabla^{2} \mathbf{v}+A^{2} \nabla \times \mathbf{b} \times \mathbf{B} \\
(\partial \mathbf{b} / \partial t) & =\nabla \times(\mathbf{v} \times \mathbf{B})+E_{m} \nabla^{2} \mathbf{b}, \\
\nabla . \mathbf{v} & =0=\nabla . \mathbf{b} .
\end{array}\right\}
$$

The quantities in (2.1) are dimensionless. The basic field $\mathbf{B}$ has been supposed to satisfy $\mathbf{B}=\nabla \Phi ; \mathbf{k}$ is the unit vector in the $z$ direction, supposed parallel to the rotation axis. A cylindrical co-ordinate system $(\varpi, \phi, z)$ and a spherical coordinate system $(r, \theta, \phi)$ are defined in the usual manner. The boundary will be denoted by $\Sigma$ and its unit normal by $\hat{\mathbf{n}}$.

The boundary conditions on $\Sigma$ are that $\mathbf{v}$ must vanish, and that $\mathbf{b}$ and

$$
\hat{\mathbf{n}} . \nabla \times\left(\mathbf{e} \cdot \hat{\mathbf{x}}_{2} \hat{\mathbf{x}}_{2}-\mathbf{e} \cdot \hat{\mathbf{x}}_{\mathbf{3}} \hat{\mathbf{x}}_{\mathbf{3}}\right)
$$

be continuous, where $\mathbf{e}$ is the electric field vector and $\hat{\mathbf{x}}_{2}$ and $\hat{\mathbf{x}}_{3}$ are mutually perpendicular unit vectors perpendicular to $\hat{n}$.

This unfamiliar form of the electromagnetic boundary conditions is convenient as it separates the electric field boundary condition into a component independent of conductivity, b. h continuous, and one depending on conductivity, equation (2.2). It is not difficult to show that the continuity of $\mathbf{e} \times \hat{\mathbf{n}}$ implies these two conditions if $\mathbf{b}$ is a function of $x_{2}, x_{3}$ and $t$. (See also Roberts 1967.) 
It is convenient to write the magnetic field in the container in terms of its poloidal and toroidal components as the electric field boundary condition in the form given involves these separately. If quantities in the container are denoted by a prime the toroidal and poloidal components may be defined by

$$
\mathbf{b}^{\prime}=\nabla \times\left(\mathbf{r} \Psi^{T}\right)+\nabla \times \nabla \times\left(\mathbf{r} \Psi^{P}\right),
$$

where the defining scalars $\Psi^{T}, \Psi^{P}$ satisfy a scalar diffusion equation with diffusion coefficient $E_{m}^{\prime}=E_{m} / S$. If $S=0$, then $\Psi^{T}=0$ and

$$
\mathbf{b}^{\prime} \rightarrow \nabla\left\{\left(r \Psi^{P}\right), r\right\} \text {. }
$$

The electric field in the fluid, scaled by $B /(4 \pi \eta L)$, is

$$
\mathbf{e}-\left(\mathbf{l} / E_{m}\right) \mathbf{v} \times \mathbf{B}+\nabla \times \mathbf{b}
$$

and it can be shown, using the equations of motion, that the electric field condition involves only the toroidal field in the container.

The complete set of boundary conditions may then be written as

$$
\left.\begin{array}{c}
\hat{\mathbf{n}} \times \mathbf{b}=\Delta_{s} \Psi^{P}, \quad \hat{\mathbf{n}} \times \mathbf{b}=\hat{\mathbf{n}} \times \nabla_{s}\left[r \Psi^{P}\right]_{, r}-\nabla_{s}\left[r \Psi^{T}\right], \\
-\left(\boldsymbol{S} / E_{m}\right) \nabla_{s} .[\mathbf{B} . \hat{\mathbf{n} v}]+S m(\mathbf{b})=2\left(r \Psi^{T}\right)_{, r \theta \phi}, \quad \mathbf{v}=0
\end{array}\right\}
$$

where $m(\mathbf{b})$ has been written for the non-cancelling current,

$$
\frac{\partial^{2}}{\partial r \partial \phi}\left(r b_{\phi}\right)-\frac{\partial^{2}}{\partial r \partial \theta}\left(r \sin \theta b_{\theta}\right)
$$

The subscript $s$ refers to horizontal co-ordinates. For example:

$$
\nabla_{s} \Phi=\hat{\boldsymbol{\theta}} \frac{1}{r} \frac{\partial \Phi}{\partial \theta}+\hat{\boldsymbol{\phi}} \frac{1}{r \sin \theta} \frac{\partial \Phi}{\partial \phi}
$$

If the fluid boundary condition, $\mathbf{v}=0$, is applied then $\nabla_{s} \cdot[\mathbf{B} . \hat{\mathbf{n} v}]$, involving only tangential derivatives of $\mathbf{v}$, will vanish as well, simplifying the electric field boundary condition.

When $E$ and $E_{m}$ are allowed to tend to zero the order of the equations (2.1) is lowered and one cannot expect to satisfy the complete set of boundary conditions. The decision as to which boundary conditions are to be retained and which discarded is not wholly trivial. Note that in this limit the order of the momentum equation, the first of (2.1), is not changed; $\nabla \times \mathbf{b}$ represents two spatial derivatives of $\mathbf{v}$ just as the discarded viscous force did. However the order of the magnetic diffusion equation is lowered.

Were $A^{2}$ to vanish, only $\mathbf{b} . \hat{\mathbf{n}}$ and $\mathbf{v} . \hat{\mathbf{n}}$ could be made to satisfy their boundary conditions. For non-zero $A^{2}$ some, but not all, of the remaining boundary conditions must be satisfied. There is no a priori way of choosing these correctly. The choice will be forced by the nature of the boundary layer. Thus a genuinely non-dissipative problem has been obtained, the correct boundary conditions for which cannot be obtained without reference to the dissipation. (By correct is meant those which are closest to physical reality; one could choose arbitrarily were dissipation never to be important.)

The limit $E_{m} \rightarrow 0, A^{2}>0$ is meaningful only if $\eta \rightarrow \infty$. If $\eta^{\prime}$ is bounded then $S$ 
vanishes and the current boundary condition determines a toroidal field in the container according to $-E_{m}^{\prime-1} \nabla_{s}$. [B.ĥt $]=2\left(r^{\top} \Psi^{T}\right)_{, r \theta \phi}$. If $\eta^{\prime} \rightarrow \infty$ in such a way that $S=S_{\infty} \neq 0$, then the current boundary condition at highest order requires that $\nabla_{s} \cdot[\mathbf{B} . \hat{\mathbf{n v}}]=\left\langle\left\langle\Psi_{, \theta \phi}^{T}\right\rangle\right\rangle$, where $\left\langle\left\langle\Psi_{, \theta \phi}^{T}\right\rangle\right\rangle$ represents the jump across the boundary. The existence of this jump defines a surface current.

The non-dissipative equations can be obtained directly from (2.1) by letting $E$ and $E_{m}$ tend to zero. The set of differential equations which results can be discussed in a general way without referring to the boundary-condition difficulties just explored.

Let the time dependence be given by $e^{i \sigma t}$. Then $\mathbf{b}$ may be eliminated simply and an equation for $\sigma$ formed by multiplying the momentum equation by the complex conjugate $\mathrm{v}^{*}$ and integrating over the volume of the fluid, viz.

$$
-\sigma^{2}\left\langle\mathbf{v}^{*}, \mathbf{v}\right\rangle+2 \sigma \operatorname{Im}\left\langle\mathbf{k} . \mathbf{v} \times \mathbf{v}^{*}\right\rangle=A^{2}\left\langle\mathbf{v}^{*} . \nabla \times \nabla \times(\mathbf{v} \times \nabla \Phi) \times \nabla \Phi\right\rangle .
$$

If $\mathbf{v}$ is proportional to $e^{i m \phi}$ then it follows from the divergence condition that its components in cylindrical co-ordinates can be written as

$$
\mathbf{v}=\mathscr{A}(u, i v, w) \exp \{i \sigma t+i m \phi\}
$$

where $\mathscr{A}$ is a complex constant and $u, v, w$ are real functions of $\varpi$ and $z$. The full right-hand side reduces to $A^{2}\left\langle\mathbf{v}^{*} . \nabla \times \nabla \times(\mathbf{v} \times \nabla \Phi) \times \nabla \Phi^{*}\right\rangle$.

Equation (2.6) is a quadratic equation for $\sigma$ and can be solved giving

$$
\sigma=-\operatorname{Im} \frac{\left\langle\mathbf{k} \cdot \mathbf{v} \times \mathbf{v}^{*}\right\rangle}{\left\langle\mathbf{v} \cdot \mathbf{v}^{*}\right\rangle}\left\{1 \pm\left[1-A^{2} \frac{\left\langle\mathbf{v}^{*} \cdot \nabla \times \nabla \times(\mathbf{v} \times \nabla \Phi) \times \nabla \Phi\right\rangle\left\langle\mathbf{v} \cdot \mathbf{v}^{*}\right\rangle}{\left[\operatorname{Im}\left\langle\mathbf{k} \cdot \mathbf{v} \times \mathbf{v}^{*}\right\rangle\right]^{2}}\right]^{\frac{1}{2}}\right\} .
$$

If the second term under the square root is small there will be two different modes. For one

$$
\sigma \approx-2 \operatorname{Im}\left\langle\mathbf{k} \cdot \mathbf{v} \times \mathbf{v}^{*}\right\rangle /\left\langle\mathbf{v} \cdot \mathbf{v}^{*}\right\rangle \text {, }
$$

which is the hydrodynamic mode (see Greenspan 1968, p. 52); for the other

$$
\sigma \approx-\frac{1}{2} \frac{\left\langle\mathbf{v}^{*} \cdot \nabla \times \nabla \times(\mathbf{v} \times \nabla \Phi) \times \nabla \Phi\right\rangle}{\left[\operatorname{Im}\left\langle\mathbf{k} \cdot \mathbf{v} \times \mathbf{v}^{*}\right\rangle\right]} A^{2}
$$

which is essentially the MAC mode of Braginskiy (1967) in the absence of buoyancy forces.

If the second term under the square root is large, two $\sigma$ of the same order result, namely

$$
\sigma \approx \pm \frac{\left|\left\langle\mathbf{v}^{*} \cdot \nabla \times \nabla \times(\mathbf{v} \times \nabla \Phi) \times \nabla \Phi\right\rangle\right|^{\frac{1}{2}}}{\left\langle\mathbf{v} \cdot \mathbf{v}^{*}\right\rangle^{\frac{1}{2}}} A
$$

The absolute value signs emphasize that $\sigma$ is real. It can be established that

$$
\begin{aligned}
\left\langle\mathbf{v}^{*} \cdot \nabla \times \nabla \times(\mathbf{v} \times \nabla \Phi) \times \nabla \Phi\right\rangle=- & \left\langle\nabla \times(\mathbf{v} \times \nabla \Phi) . \nabla \times\left(\mathbf{v}^{*} \times \nabla \Phi\right)\right\rangle \\
& \quad+\int_{\text {surpace }}(\hat{\mathbf{n}} \cdot \nabla \Phi) \mathbf{v}^{*} \cdot\{(\nabla \Phi . \nabla) \mathbf{v}-(\mathbf{v} \cdot \nabla) \nabla \Phi\} .
\end{aligned}
$$

If the outside of the volume is extended beyond the fluid then the surface integral vanishes and $\sigma$ is real.

Equation (2.10) can hold under two circumstances: if $A^{2}$ is larger than order 
unity, or if $\operatorname{Im}\left\langle\mathbf{k} . \mathbf{v} \times \mathbf{v}^{*}\right\rangle$ is very much smaller than $A$. In the former circumstance the perturbation analysis envisioned is not applicable; this possibility will not be considered further. The second possibility is of greater interest as the modes represented are like the usual geostrophic modes. In a sphere these represent solid rotation of the interior at a rate different from that of the container, as well as more complicated motions sharing a purely azimuthal velocity depending only on the cylindrical radial co-ordinate $\varpi$.

This mode is of interest as it is the mode participating in any spin-up process and its analysis will shed some more light on the hydromagnetic spin-up problem, so far attacked only in an infinite domain (Loper \& Benton 1970; Benton \& Loper 1969). Its excitation in a geophysical context is implied by the irregular changes in the length of the day (Munk \& MacDonald 1960), which clearly imply interchange of angular momentum between the core and the mantle.

The relations (2.8)-(2.10) define three cases. These cases will be labelled in descending order of magnitude of the leading term of $\sigma$. Case I corresponds to equation (2.8), case II to (2.10) and case III to (2.9). The force balances involved are: inertial-Coriolis (the usual inertial wave), inertial-Lorentz and CoriolisLorentz. The nomenclature is summarized in table 1.

For all three cases the existence of an asymptotic expansion will be supposed. That is, $\mathbf{v}, \mathbf{b}, \Pi$ and $\sigma$ can be expanded in a formally infinite series of the type

$$
\mathbf{v}=\sum_{j=i}^{\infty} \epsilon^{(j)}\left[\mathbf{v}^{(j)}+\tilde{\mathbf{v}}^{(j)}\right]
$$

The set $\left\{\epsilon^{(j)}\right\}$ is to be determined, subject to the requirement that $\epsilon^{(j+i)} / \epsilon^{(j)} \ll 1$; $\epsilon^{(0)}=1$. Functions distinguished by a tilde are boundary-layer functions; these will be explored more fully in $\S 3$ below.

The lowest order interior solution in each case satisfies a dissipationless pair of vector equations. These may be written as

$$
\begin{aligned}
& \left.\begin{array}{c}
i \sigma^{(0)} \mathbf{v}^{(0)}+2 \mathbf{k} \times \mathbf{v}^{(0)}+\nabla \Pi^{(0)}=0, \\
i \sigma^{(0)} \mathbf{b}^{(0)}=\nabla \times\left(\mathbf{v}^{(0)} \times \nabla \Phi\right), \quad(\sigma \sim 1) ;
\end{array}\right\} \\
& \left.\begin{array}{c}
2 \mathbf{k} \times \mathbf{v}^{(1)}+\nabla \Pi^{(1)}=0, \\
i \sigma^{(1)} \mathbf{b}^{(0)}=\nabla \times\left(\mathbf{v}^{(\mathbf{l})} \times \nabla \Phi\right), \quad(\sigma \sim A) ;
\end{array}\right\} \\
& \left.\begin{array}{c}
2 \mathbf{k} \times \mathbf{v}^{(2)}+\nabla \Pi^{(2)}=\nabla \times b^{(0)} \times \nabla \Phi, \\
i \sigma^{(2)} \mathbf{b}^{(0)}=\nabla \times\left(\mathbf{v}^{(2)} \times \nabla \Phi\right), \quad\left(\sigma \sim A^{2}\right) .
\end{array}\right\}
\end{aligned}
$$

The stated force balances are obvious in cases I and III. For case II the force balance does not appear explicitly and one must go to the integrated equation (2.6) to see the force balance. If $\sigma \sim A$ and $2 \operatorname{Im}\left\langle\mathbf{k} . \mathbf{v} \times \mathbf{v}^{*}\right\rangle \ll A$ then the second term in (2.6) will be small compared to the others and the balance will be, as stated, between the inertial force represented by the first term and the Lorentz force represented by the right-hand side. 


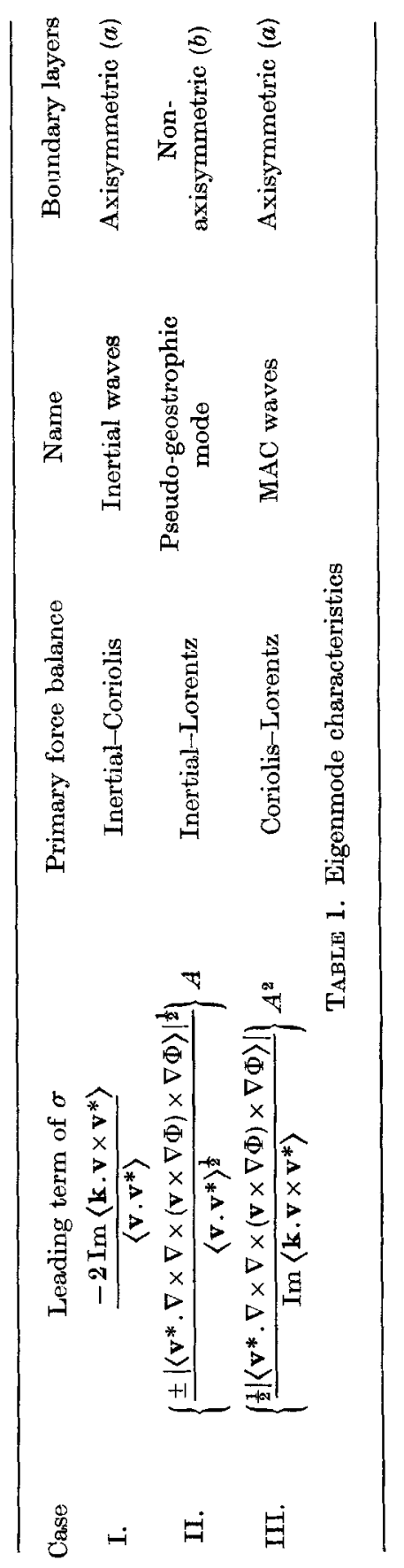




\section{The boundary layers}

In this section the general boundary-layer structure in a sphere in the presence of an imposed magnetic field is developed. Since boundary-layer phenomena are essentially local it is not necessary to restrict severely the imposed magnetic field; a more general magnetic field than that taken in $\$ 2$ may be used. The only crucial point is that of axisymmetry. To develop boundary layers appropriate to cases I and III it is necessary that the basic field, B, be axisymmetric. For case II there is no content unless $\mathbf{B}$ is not axisymmetric.

The analysis is similar for each circumstance. Cases I and III involve axisymmetric boundary layers, which will be referred to as type $(a)$, and case II involves non-axisymmetric boundary layers, which will be referred to as type (b). In this section $\tilde{\mathbf{v}}, \tilde{\mathbf{b}}$ and $\tilde{I}$ will be used to denote the leading term of each boundary-layer expansion, primarily for aesthetic reasons. Where a distinction is necessary a superscript $(a)$ or $(b)$ will be used to distinguish between the two types of boundary-layer phenomena.

The usual boundary-layer hypothesis will be adopted (cf. Greenspan 1968, pp. $23 \mathrm{ff}$.) from which, using $\delta$ as the 'boundary-layer thickness', the boundarylayer version of the second of (2.1) can be seen to be, to leading order,

$$
\left.\begin{array}{l}
{\left[i \sigma-E_{m} \delta^{-2}\right] \tilde{\mathbf{b}} \cdot \hat{\mathbf{n}}=-\nabla_{s} \cdot\left(B_{r} \tilde{\mathbf{v}}\right),} \\
{\left[i \sigma-E_{m} \delta^{-2}\right] \tilde{\mathbf{b}}_{s}=-(\mathrm{l} / \delta) B_{r} \tilde{\mathbf{v}}_{s},}
\end{array}\right\}
$$

where the subscript $s$ denotes vectors lying parallel to the surface. For any vector $\mathbf{a}: \mathbf{a}=\mathbf{a} \cdot \hat{\mathbf{n}} \hat{\mathbf{n}}+\mathbf{a}_{s} . B_{r}$ is the radial component of the imposed magnetic field. The equations (3.1) are valid only for regions where $\left|B_{r}\right| \gg\left|\delta \mathbf{B}_{s}\right|$, so one might expect incorrect conclusions to be drawn if the imposed field is parallel to the boundary. However, a similar analysis (Gans 1971) has shown that the influence of a magnetic field parallel to the boundary on the viscous boundary layers is negligible. The phenomena with which this paper is concerned, and the novel properties of the boundary layers, depend critically on the existence of coupling between magnetic and viscous boundary layers so that a magnetic field which exerts a negligible influence on the viscous boundary layer is unimportant. (It can be remarked in passing that in the earth $|\delta|$ will not exceed $10^{-4}$, and so even a toroidal field of $1000 \mathrm{G}$ will not be important compared to the characteristic poloidal field of $5 \mathrm{G}$ at the core-mantle boundary.)

As the size of $\delta$ is unknown, it is never appropriate to neglect the Lorentz force or the time derivative of the velocity in the momentum equation. One must retain the leading contribution of each individual term. The normal and tangential components of the type $(a)$ boundary-layer momentum equations are then

$$
\left.\begin{array}{l}
{\left[i \sigma-E \delta^{-2}\right] \tilde{\mathbf{v}} \cdot \hat{\mathbf{n}}+2 \mathbf{k} \times \tilde{\mathbf{v}} \cdot \hat{\mathbf{n}}+\tilde{\Pi} / \delta=\left(A^{2} / \delta\right) \mathbf{B} \cdot \tilde{\mathbf{b}}_{s},} \\
{\left[i \sigma-E \delta^{-2}\right] \tilde{\mathbf{v}}_{s}+2 \mathbf{k} \times \tilde{\mathbf{v}}_{s}+\nabla_{s} \tilde{\Pi}=\left(A^{2} / \delta\right) B_{r} \tilde{\mathbf{b}}_{s} .}
\end{array}\right\}
$$

The balance in the first equation is between the pressure and the Lorentz force, giving $\tilde{\Pi}=A^{2} \mathbf{B} \cdot \tilde{\mathrm{b}}_{s}$. Substitution of this value into the second equation shows that the pressure term is negligible compared to the Lorentz force. This is 
analogous to the usual boundary-layer result that pressure terms are negligible in the boundary-layer equations.

The type $(b)$ boundary-layer equations are somewhat more complex. From (3.1) it can be seen that $\tilde{\mathbf{b}}$ is 'singly periodic' in the azimuthal co-ordinate for both type $(a)$ and type $(b)$ systems. Thus the $(b)$ boundary-layer Lorentz force has both a rectified (axisymmetric) and a 'doubly-periodic' component. The doubly-periodic component does not give rise to a doubly-periodic $\widetilde{\mathbf{v}}^{(b)}$ but instead makes $\delta^{(b)}$ a function of azimuth as well as co-latitude.

The type $(a)$ boundary-layer equations are derived by combining the horizontal components of (3.1) and (3.2) into a single equation in $\tilde{\mathbf{v}}_{s}$, viz.

$$
\left\{i \sigma-E / \delta^{2}+A^{2} B_{r}^{2} /\left(i \sigma \delta^{2}-E_{m}\right)\right\} \tilde{\mathbf{v}}_{s}+2 \mathbf{k} \times \widetilde{\mathbf{v}}_{s}=0 .
$$

The boundary-layer thicknesses are then obtained from

$$
\sigma(\sigma \pm 2 \cos \theta) \delta^{4}+\left[i(\sigma \pm 2 \cos \theta) E_{m}+i \sigma E+A^{2} B_{r}^{2}\right] \delta^{2}-E E_{m}=0 .
$$

To deduce the type $(b)$ boundary-layer structure it is necessary to be more specific regarding the dependence of $B_{r}$ on azimuth. To that end let $B_{r}^{(b)}$ be proportional to $\exp [i m \phi]$. The horizontal component of (3.1) is then

$$
\left(i \sigma-E_{m} \delta^{-2}\right) \tilde{\mathrm{b}}^{(b)}=(1 / \delta) \beta e^{i m \phi} \tilde{\mathbf{v}}^{(b)},
$$

where $\beta=\beta(\theta)$ represents the non-azimuthal variation of $B_{r}^{(b)}$ in the vicinity of the boundary. The use of complex notation means that the physical Lorentz force in the type $(b)$ boundary-layer equations is

$$
\frac{1}{2} A^{2} \beta\left(e^{i m \phi}+e^{-i m \phi}\right)\left[\left(\tilde{\mathbf{b}}^{(b)} / \delta\right)+\left(\tilde{\mathbf{b}}^{(b) *} / \delta^{*}\right)\right],
$$

so that the right-hand side of (3.2) should be written as

$$
A^{2} \beta \cos (m \phi) \tilde{\mathrm{b}}^{(b)} / \delta \text {. }
$$

Thus the result of these manipulations is that the type $(b)$ boundary-layer parameter $\delta^{(b)}$ obeys (3.4) with $B_{r}^{2}$ replaced by $\beta\left(\cos ^{2} m \phi+i \sin m \phi \cos m \phi\right)$. This quantity will be denoted by $\left(B_{r}^{2}\right)^{(b)}$ if the meaning of $B_{r}^{2}$ is not clear from the context.

Writing out the type (b) boundary-layer equation specifically,

$$
\sigma(\sigma \pm 2 \cos \theta) \delta^{(b) 4}+\left[i(\sigma \pm 2 \cos \theta) E_{m}+i \sigma E+A^{2}\left(B_{r}^{2}\right)^{(b)}\right] \delta^{(b) 2}-E E_{m}=0
$$

and one notes that the coefficient of $\delta^{(b) 4}$ is independent of azimuth, as is the constant term. Thus the dependence of $B_{r}$ on azimuth does not introduce additional singularities of the type represented by $\sigma \pm 2 \cos \theta=0$. Because of this the limiting properties of both type $(a)$ and type $(b)$ boundary layers, viewed as functions of $E, E_{m}, A^{2}, \sigma$ and $\theta$, will be essentially the same and a single investigation of such properties can be made.

Equation (3.4) can be solved for $\delta^{2}$ directly. There are then eight possible values of $\delta$, four of which are eliminated by the requirement that $R e \delta<0$. The remaining four come in pairs, corresponding to the northern and southern hemisphere expressions. 


\section{Let}

$$
\begin{aligned}
& x=E, \quad y=(\sigma \pm 2 \cos \theta) / \sigma E_{m}, \quad z=A^{2} B_{r}^{2} / \sigma \\
& \left.g=(\sigma \pm 2 \cos \theta) \delta^{2}=-\frac{1}{2}(i x-i y+z) \pm \frac{1}{2} f^{\frac{1}{2}},\right\} \\
& f=(i x-i y+z)^{2}-4 x y \text {. }
\end{aligned}
$$

and define

It will be convenient to assign numbers to the boundary layers according to the hemisphere in which the potential singularity (reckoning $\sigma>0$ ) will occur and according to the role, viscous or magnetic, the boundary layer plays. The northern hemisphere singularity will be associated with the numbers 1 and 3 and the southern hemisphere singularity with 2 and 4 . This is summarized in table 2 . The viscous boundary layer ( 1 and 2 ) is that in which the major velocity corrections take place and the magnetic boundary layer (3 and 4) is that in which the major magnetic field corrections take place. This will be demonstrated below.

$\begin{array}{ccc}\text { Boundary-layer number } & \text { Role } & \text { Hemisphere of singularity } \\ \mathbf{1} & \text { Viscous } & \text { Northern } \\ \mathbf{2} & \text { Viscous } & \text { Southern } \\ \mathbf{3} & \text { Magnetic } & \text { Northern } \\ 4 & \text { Magnetic } & \text { Southern }\end{array}$

TABLE 2. The boundary layers

There are simple representations of $g$ if $x, y$ or $z$ vanishes identically. These represent inviscid, infinitely conducting and vanishing magnetic field, respectively. For these three subsets of parameter space

$$
g(0, y, z)=\left\{\begin{array}{c}
0 \\
i y-z
\end{array} ; \quad g(x, 0, z)=\left\{\begin{array}{c}
-i x-z \\
0
\end{array} ; \quad g(x, y, 0)=\left\{\begin{array}{c}
-i x \\
i y
\end{array} .\right.\right.\right.
$$

The neighbourhoods of these exact solutions can be investigated by supposing that $x, y, z$ are in turn very much smaller than the other two and expanding $f^{\frac{1}{2}}$ in a Taylor series. The neighbourhoods are numbered I, II, III. To first order in each small parameter

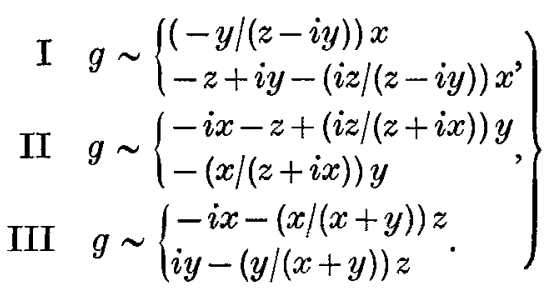

As before the upper member of each pair is the viscous member and the lower the magnetic. The third region illustrates, for small $z$, the process that moves the linear term in $z$ from the magnetic to the viscous boundary layer in going from region I to region II. This is an unexpected development which may be interpreted in terms of Alfvén-like waves.

Since $z$ is real it can lead to wave-like behaviour. To see this one need merely find $\delta$ for the exact solutions represented by (3.8). Taking the square root gives

$$
\begin{aligned}
\delta=-\left\{\frac{1}{2}\left[\left(a^{2}+z^{2} /(\sigma \pm 2 \cos \theta)^{2}\right)^{\frac{1}{2}}+z /(\sigma \pm 2 \cos \theta)\right]\right\}^{\frac{1}{2}} \\
-i \operatorname{sgn}(a)\left\{\frac{1}{2}\left[\left(a^{2}+z^{2} /(\sigma \pm 2 \cos \theta)^{2}\right)^{\frac{1}{2}}-z /(\sigma \pm 2 \cos \theta)\right]\right\}^{\frac{1}{2}},
\end{aligned}
$$


where $a$ stands for either $E_{m} / \sigma$ or $E /(\sigma \pm 2 \cos \theta)$. The $\operatorname{symbol} \operatorname{sgn}(a)=1$ if $a>0$ and -1 if $a<0$. A purely imaginary $\delta$ represents waves in the radial direction. The potentiality for this arises when $|z /(\sigma \pm 2 \cos \theta)|$ is large compared to $a$, and $z /(\sigma \pm 2 \cos \theta)<0$.

In the two exact solutions given by (3.8) this wave is always associated with the dissipative term. In the doubly dissipative system it is associated with the larger dissipative parameter, the flow adjusting itself to ensure the maximum decay of these boundary-layer waves by minimizing the ratio $|\operatorname{Im} \delta / \operatorname{Re} \delta|$. It is finally to be remarked that the waves are Alfvén-like since their exponential dependence is like $\exp [i(\sigma t+r / A)]$. Re-dimensionalizing this equation and using the Alfvén speed $V_{A}=B /(4 \pi \rho)^{\frac{1}{2}}$ one obtains $\exp \left[i \omega\left(\sigma t+r / V_{A}\right)\right]$, representing Alfvén waves with wave-number $\sigma^{-1}$.

It will now be established that the viscous boundary layer is that in which the major velocity corrections are made and that the magnetic boundary layer is that in which the major magnetic field corrections are made.

This is most easily done by considering the factor relating $\tilde{\mathbf{b}}$ and $\tilde{\mathbf{v}}$, derived from (3.1), viz.

$$
\tilde{\mathbf{b}}=-B_{r} \delta /\left(i \sigma \delta^{2}-E_{m}\right) \tilde{\mathbf{v}}=T \tilde{\mathbf{v}} .
$$

For each boundary layer there is a constant $T_{p}$ related to the individual boundary layer by

$$
T_{p}=-B_{r} \delta_{p} /\left(i \sigma \delta_{p}^{2}+E_{m}\right)
$$

The ratio $T_{\text {v1scous }} / T_{\text {magnetic }}$ should be small compared to unity. Investigation of the ratio $T_{1} / T_{3}$ in all regions suffices to establish the truth of this. In terms of $x, y, z$ and $g$ one obtains

$$
\frac{T_{1}}{T_{3}}=\frac{\delta_{1}}{\delta_{3}} \frac{i \sigma \delta_{3}^{2}+E_{m}}{i \sigma \delta_{1}^{2}+E_{m}}=\left(\frac{g_{1}}{g_{3}}\right)^{\frac{1}{2}} \frac{i g_{3}+(\sigma-2 \cos \theta) E_{m}}{i g_{1}+(\sigma-2 \cos \theta) E_{m}} .
$$

By using the representations (3.9) this ratio can be evaluated. The results to leading order are summarized in table 3 from which it can be seen that $T_{1} / T_{3}$ is indeed small. (For those entries for which $g_{3}=i y$ the denominator of $T_{3}$ would vanish. For these, therefore, $-\sigma\left[B_{r} /(\sigma-2 \cos \theta)^{\frac{1}{2}}\right] T_{3}$ has been calculated including the next term of $g_{3}$ in each case.)

If $\sigma \sim A^{2}$ two boundary conditions are satisfied by the interior solution and not all the boundary layers are necessary. It was remarked that the boundarylayer analysis would indicate what boundary condition the interior solution must satisfy.

It is first to be noted that if $\sigma \sim A^{2}, z \sim 1$. The quantity $y$ remains small as $E_{m} / A^{2} \ll 1$ was originally assumed. Regions I and II are both pertinent, and it can be seen from equation (3.8) that the fatter boundary layer has a thickness of order unity which conflicts with the boundary-layer hypothesis. Thus the fatter boundary layer in each region must be rejected. In region $I$ the magnetic boundary layer must be rejected, in region II the viscous boundary layer must be rejected. However, rejection of a given boundary layer does not necessarily imply rejection of the corresponding boundary condition. To make a decision regarding boundary conditions it is necessary to examine the boundary-layer functions. 


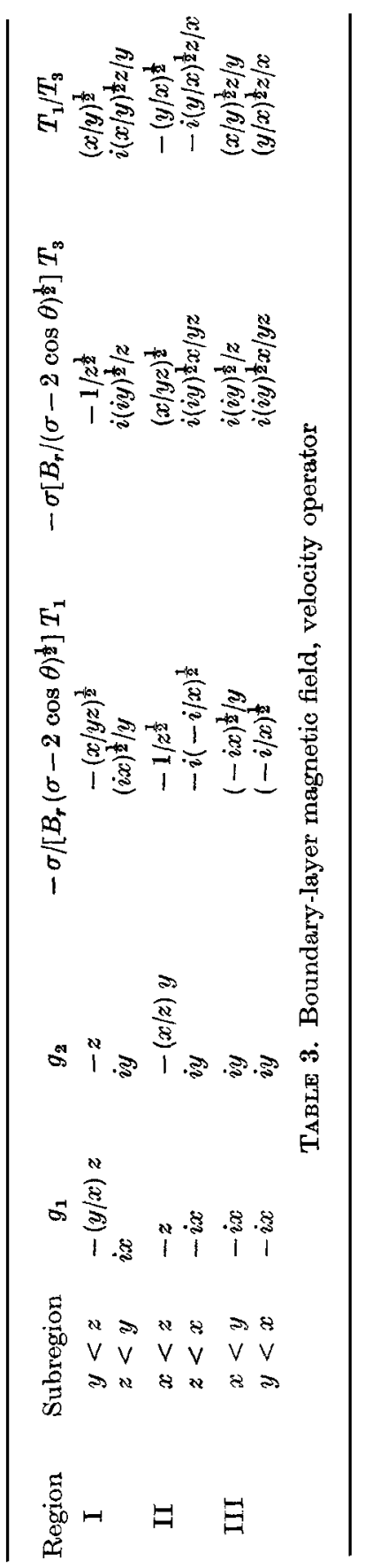


For the remaining boundary layer to satisfy the magnetic boundary conditions it is necessary that $\vec{b}$ be of order unity (see $(2.11 c)$ ); to satisfy the viscous boundary conditions it is necessary that $\tilde{\mathbf{v}}$ be of order $\epsilon^{(2)}$, which can be approximated by $A^{2}$ for the sake of discussion. If the boundary-layer solution is to separate cleanly from the non-dissipative solution the boundary-layer quantity not being matched must be smaller than its non-dissipative counterpart. If $\tilde{\mathbf{b}}$ is of order unity, $\tilde{\mathbf{v}}$ must be smaller than order $\epsilon^{(2)}$. If $\tilde{\mathbf{v}}$ is of order $\epsilon^{(2)}, \tilde{\mathbf{b}}$ must be smaller than order unity.

In region $I$ the viscous boundary layer is retained. The relation between the orders of magnitude of $\tilde{\mathbf{v}}$ and $\tilde{\mathrm{b}}$ is given by examination of $T_{1}$. From table 3

$$
\tilde{\mathbf{b}} \sim(x / y z)^{\frac{1}{2}}\left(B_{r}(\sigma-2 \cos \theta)^{\frac{1}{2}} / \sigma\right) \tilde{\mathbf{v}} .
$$

To satisfy magnetic boundary conditions $\tilde{b}$ will be of order unity and, to make $\tilde{\mathbf{v}}$ smaller than order $\epsilon^{(2)}, T_{1}>1 / \epsilon^{(2)}$. To satisfy viscous boundary conditions $\tilde{\mathbf{v}}$ must be of order $e^{(2)}$ and $T_{1}<1 / \epsilon^{(2)}$. In region I the boundary condition criterion is the size of

$$
\epsilon^{(2)}(x / y z)^{\frac{1}{2}}\left(B_{r}(\sigma-2 \cos \theta)^{\frac{1}{2}} / \sigma\right)=\epsilon^{(2)}\left\{E / E_{m} A^{2}\right\}^{\frac{1}{2}} B_{r}=B_{r} K .
$$

Because the remaining boundary layer in region II has the same proportionality constant, $(x / y z)^{\frac{1}{2}}$, the same criterion will be derived. It can be written in terms of the physical constants using $\epsilon^{(2)} \sim A^{2}$ to simplify the expression. One obtains

$$
K=\left[E A^{2} / E_{m}\right]^{\frac{1}{2}}=\left[B^{2} / 4 \pi \rho \omega^{2} L^{2}\right]^{\frac{1}{2}}(4 \pi \eta \nu)^{\frac{1}{2}}=\left(V_{A} / \omega L\right) \operatorname{Pr}_{m}^{\frac{1}{2}},
$$

where $\operatorname{Pr}_{m}$ is the magnetic Prandtl number.

For liquid metals the magnetic Prandtl number $4 \pi \eta \nu \ll 1$. Typical values are $10^{-6}$. Thus $K$ will be small, making the viscous boundary conditions appropriate everywhere.

\section{Non-dissipative exploration of the pseudo-geostrophic mode}

In this section the consequences of a purely non-dissipative expansion of the case II mode will be discussed. The small parameters $\varepsilon^{(j)}=A^{j}$ and the initial expansion is then straightforward. The initial velocity $\mathbf{v}^{(1)}$ is the usual geostrophic velocity in a sphere given by

$$
\mathbf{v}^{(1)}=v(\varpi) \hat{\boldsymbol{\phi}}
$$

and the associated magnetic field and shifted (hence non-zero) frequency are coupled through the relation

$$
i \sigma^{(\mathbf{1})} \mathbf{b}^{(0)}=\nabla \times\left(\mathbf{v}^{(\mathbf{1})} \times \nabla \Phi\right) .
$$

If $\Phi \propto e^{i m \phi}$ the first non-dissipative interation in the momentum equation leads to

$$
i \sigma^{(1)} \mathbf{v}^{(1)}+2 \mathbf{k} \times \mathbf{v}^{(2)}+\nabla \Pi^{(2)}=\frac{1}{2} \nabla \times \mathbf{b}^{(0)} \times\left[\nabla \Phi+\nabla \Phi^{*}\right] .
$$

The eigenfrequency $\sigma^{(1)}$ can be calculated as follows. Multiply equation (4.3) by $\mathbf{v}^{(1) *}=v(\omega) \exp \left\{-i \sigma^{(1)} t\right\} \hat{\boldsymbol{\phi}}$ and integrate over the volume of the fluid. Multiply the relation conjugate to the first of $(2.11 b)$, viz.

$$
2 \mathbf{k} \times v(\varpi) \hat{\boldsymbol{\phi}} \exp \left\{-i \sigma^{(\mathbf{1})} t\right\}+\nabla \Pi^{*}=0,
$$


by $\mathbf{v}^{(2)}$ and integrate over the volume of the fluid. Since $\mathbf{v}^{(2)} \cdot \hat{\mathbf{n}}=0$ in the nondissipative limit the pressure terms integrate to zero in both equations, leaving the pair

$$
\left.\begin{array}{c}
i \sigma^{(1)}\left\langle[v(\varpi)]^{2}\right\rangle+2\left\langle\mathbf{v}^{(1) *} . \mathbf{k} \times \mathbf{v}^{(2)}\right\rangle=\frac{1}{2}\left\langle\mathbf{v}^{(1) *} \cdot\left[\nabla \times \mathbf{b}^{(0)} \times\left(\nabla \Phi+\nabla \Phi^{*}\right)\right]\right\rangle, \\
2\left\langle\mathbf{v}^{(2)} \cdot \mathbf{k} \times \mathbf{v}^{(1) *}\right\rangle=0 .
\end{array}\right\}
$$

Using this pair one can derive an expression for $\sigma^{(\mathbf{1})}$. After eliminating $\mathbf{b}^{(0)}$ and using (4.2) the result is

$$
\left[\sigma^{(\mathbf{1})}\right]^{2}=-\frac{1}{2}\left\langle v(\varpi) \hat{\boldsymbol{\phi}} \cdot\left[\nabla \times \nabla \times(v(\varpi) \hat{\boldsymbol{\phi}} \times \nabla \Phi) \times \nabla \Phi^{*}\right]\right\rangle /\left\langle[v(\varpi)]^{2}\right\rangle .
$$

The particular case $v(\varpi)=\varpi$ is of interest because it represents a potential spin-up process and because such a difference in rotation rate between sphere and container must arise from conservation of angular momentum during the observed irregular changes in the length of the day. However the strictly nondissipative system under consideration does not admit such a solution, as can be easily shown. By direct calculation

$$
\nabla \times(\varpi \hat{\phi} \times \nabla \Phi)=-\nabla \Phi, \phi .
$$

Thus the curl of $\mathbf{b}^{(0)}$ vanishes and the numerator on the right-hand side of (4.6) vanishes. This is a singular result since $\sigma^{(1)} b^{(0)}$ does not vanish. The conclusion one reaches is that $\nabla^{(1)}=\varpi \hat{\phi} \exp \left[i \sigma^{(1)} t\right]$ is not an admissable solution in the absence of dissipation.

Physically the contradiction can be seen as follows. In the non-dissipative system the field lines are frozen into the fluid. The lack of axisymmetry means that work is being done by the field if the interior is made to rotate at a different rate from the container. The absence of dissipation means that the propagation time of this information is zero, so that the mode 'decays' instantaneously. In a physical system in which dissipation exists this contradiction is resolved, this is discussed in $\S 5$ below.

\section{The basic pseudo-geostrophic mode: dissipation}

In this section the dissipative expansion, involving boundary-layer terms, mentioned in $\$ 2$ will be considered for the special case for which the leading term in the velocity is $\varpi \hat{\boldsymbol{\phi}} \exp \left[i \sigma^{(\mathbf{1})} t\right]$. This is a subset of case II and the leading terms of $\mathbf{v}$ and $\mathbf{b}$ are of order $\epsilon^{(\mathbf{I})}$ and unity, respectively. It will be supposed that $\epsilon^{(1)} \gg A^{2}$ and the conditions under which this is true will be given below. Here a tilde is being used to denote boundary-layer functions. The hierarchy of problems to be solved in sequence is: (i) the non-dissipative geostrophic problem; (ii) the boundary-layer problem (3.6); (iii) the solvability conditions for the secondary non-dissipative problem.

(Divergence of flow from the boundary layer will give rise to an efflux $\tilde{\mathbf{v}}^{(2)} \cdot \hat{\mathbf{n}}$, given by the integral of $\nabla_{s} .\left(\tilde{\mathbf{v}}^{(1)}\right)$, from the boundary into the interior. In order to maintain the condition $\mathbf{v} . \hat{\mathbf{n}}=\mathbf{0}$ on the boundary a secondary non-dissipative flow $\mathbf{v}^{(2)}$ must be found such that $\left(\mathbf{v}^{(2)}+\tilde{\mathbf{v}}^{(2)}\right)$. $\hat{\mathbf{n}}$ vanishes on the boundary. The condition that the problem determining $\mathbf{v}^{(2)}$ has a solution determines $\epsilon^{(1)} \sigma^{(1)}$, 
which determines both the frequency and decay rate of the mode. In particular $1 /\left[\epsilon^{(1)} \sigma^{(1)}\right]$ is the dimensionless spin-up time under the conditions envisioned.)

The solvability condition is obtained in the same manner as (4.6). In the dissipative case a term appears on the right-hand side of the second of (4.5) because $\mathbf{v}^{(2)}$ by itself does not satisfy the normal boundary condition on the velocity. The Lorentz force term vanishes as before and one finally obtains an expression for $\epsilon^{(1)} \sigma^{(1)}$ in terms of the boundary-layer divergence, viz.

$$
\epsilon^{(1)} \sigma^{(1)}=\left(\frac{-i}{\left\langle v^{(1) *} \cdot v^{(1)}\right\rangle}\right) \int_{\text {surface }}\left\{\int \nabla \cdot \tilde{\mathbf{v}}^{(1)} d n\right\} d \Sigma,
$$

which expresses the expected result that $\epsilon^{(1)}$ is of the order of the boundarylayer thickness.

This procedure is analogous to that used in the usual fluid dynamic problems (c.f. Greenspan 1968) and has the same pitfalls as the hydrodynamic procedure. It is expected that the results will be equally good. The goal of this section is to construct an estimate of the right-hand side of (5.1) taking into account all the non-dimensional parameters, including $S$. This is a non-trivial task which can be divided into several stages.

The first stage is to find $\tilde{\mathbf{v}}^{(1)}$ and $\tilde{\mathbf{b}}^{(0)}$. This involves the simultaneous calculation of the toroidal field in the container, as that field is coupled to the boundarylayer conditions. The toroidal defining scalar in the container $\Psi^{2} T$ cannot be found in closed form and must be estimated. This is accomplished by eliminating everything except $\Psi^{T}$ from the boundary conditions by using the continuity of $\mathbf{v}_{s}$ and $\mathbf{b}_{s}$. The electric field boundary condition reduces to a second-order differential equation for $\Psi^{T}$ as a function of $\theta$ and $\phi$ from which the magnitude of $\Psi^{* T}$ may be estimated.

Having found $\tilde{\mathbf{v}}^{(1)}, \tilde{\mathrm{b}}^{(0)}$ and $\Psi^{T}$ one must then form $\nabla_{s} \cdot \tilde{\mathbf{v}}^{(1)}$, integrate in the normal direction to obtain $\hat{\mathbf{n}} . \tilde{\mathbf{v}}^{(2)}$, multiply by $\Pi^{*}=\varpi^{2} \exp \left[-i \sigma^{(1)} t\right]$ and integrate over the surface. Because of the involved dependence of the boundary-layer quantities $\left\{\delta_{p}\right\}$ on $\theta$ and $\phi$ this cannot be done analytically and one is restricted to construc. tion of an estimate.

The details are straightforward but tedious. In order to reduce the paper to a manageable length it has been necessary to suppress all but the most important points. (A more detailed exposition is available directly from the author.)

The continuity of $\mathbf{v}_{s}$ and $\mathbf{b}_{s}$ must be considered simultaneously. Even though $\tilde{\mathbf{v}}_{s}$ is large in the viscous boundary layer and $\tilde{b}_{s}$ in the magnetic the contribution of all four terms must be considered in all four boundary conditions. If

$$
\left.\begin{array}{l}
\tilde{\mathrm{v}}^{(\mathbf{1})}=\sum_{p=1}^{4} A_{p}\left[\hat{\boldsymbol{\theta}}+(-)^{p} i \hat{\boldsymbol{\phi}}\right] \exp \left[(1-r) / \delta_{p}+i A \sigma^{(\mathbf{1})} t\right], \\
\tilde{\mathrm{b}}^{(\mathbf{0})}=\epsilon^{(\mathbf{1})} \sum_{p=1}^{4} T_{p} A_{p}\left[\hat{\boldsymbol{\theta}}+(-)^{p} i \hat{\boldsymbol{\phi}}\right] \exp \left[(1-r) / \delta_{p}+i A \sigma^{(1)} t\right],
\end{array}\right\}
$$

then the $A_{p}$ are given by

$$
\left.\begin{array}{l}
A_{1}=-\frac{1}{2}\left(T_{3} i \varpi+B^{+} / \epsilon\right)\left(T_{3}-T_{1}\right)^{-1}, \quad A_{3}=\frac{1}{2}\left(T_{1} i \varpi+B^{+} / \epsilon\right)\left(T_{3}-T_{1}\right)^{-1} \\
A_{2}=\frac{1}{2}\left(T_{4} i \varpi-B^{-} / \epsilon\right)\left(T_{4}-T_{2}\right)^{-1}, \quad A_{4}=-\frac{1}{2}\left(T_{2} i \varpi-B^{-} / \epsilon\right)\left(T_{4}-T_{2}\right)^{-1},
\end{array}\right\}
$$

where

$$
B^{ \pm}=(\hat{\boldsymbol{\theta}} \pm i \hat{\boldsymbol{\phi}}) \cdot\left(\mathbf{b}_{\mathbf{s}}^{(0)^{\prime}}-\mathbf{b}_{\mathbf{s}}^{(0)}\right) \exp \left[-i \epsilon^{(1)} \sigma^{(1)} t\right]
$$


The last boundary condition, given by the last of (2.7), is in the boundarylayer limit

$$
\epsilon^{(1)} S \sum_{p=1}^{4} \frac{\partial}{\partial \theta}\left(\sin \theta \frac{T_{p} A_{p}}{\delta_{p}}\right)+(-)^{p} m \frac{T_{p} A_{p}}{\delta_{p}}=-2\left(r \Psi^{\cdot T}\right)_{, r \theta \phi} .
$$

The boundary conditions for (5.5) are that $\Psi^{T}=0$ at $\theta=0, \pi$.

A general solution for $\Psi^{T}$ satisfying (2.4) can be written down directly, in the form of an infinite series. The infinite series in $n$ must then be truncated to allow one to proceed. The inhomogeneous terms (with respect to $\Psi^{T}$ as the variable), in (5.5) may be either odd or even functions of $\cos \theta$ so that both odd and even contributions to $\Psi^{\top}$ are possible. The simplest useful truncation, then, is to consider two terms $n=m$ and $n=m+1$.

To effect the separation (5.5) is multiplied in turn by $P_{m}^{m}(\cos \theta) e^{-i m \phi}$ and $P_{m+1}^{m}(\cos \theta) e^{-i m \phi}$ and integrated over $0 \leqslant \phi \leqslant 2 \pi$ and $0 \leqslant \theta \leqslant \pi$. In performing the $\phi$ integration the quantity $\left(B_{r}^{2}\right)^{(b)}$ appearing in the $\delta_{p}$ is taken to be a constant equal to its average value.

Estimation of the $\theta$ integrals can be effected most easily using the symmetry properties of the $A_{p}$ and $B^{ \pm}$. When $B_{r}$ is an odd function of $\cos \theta$ it can be shown that $\Psi^{T}$ must be an even function of $\cos \theta$. After considerable manipulation the following order-of-magnitude equation may be written:

$$
\int\left(2 \Lambda-S G_{2}\right) D_{m m} \sim \int G_{1}
$$

where

$$
\left.\begin{array}{l}
G_{1}=\epsilon^{(1)} B_{r} /\left(E_{m}+i\left[\sigma E E_{m} /(2 \mu-\sigma)\right]^{\frac{1}{2}}\right), \\
G_{2}=i \sigma\left(\bar{\delta}_{1}+\bar{\delta}_{3}\right) /\left(E_{m}+i\left[\sigma E E_{m} /(2 \mu-\sigma)\right]^{\frac{1}{2}}\right),
\end{array}\right\}
$$

$D_{m m}$ is the coefficient of the $\Psi^{T}, \sigma$ has been written for $\epsilon^{(1)} \sigma^{(1)}$ and $\Lambda$ represents the normal derivative.

In performing the integration the contribution from the singular region $2 \mu \sim \sigma$ is negligible. After integration one obtains

$$
D_{m m} \sim\left(\epsilon^{(1)} S / N_{m}\right) /\left(\alpha S\left(\sigma A^{2}\right)^{\frac{1}{2}}+\Lambda E_{m}\right),
$$

where $N_{m}=1,3,5, \ldots(2 m-1)$ and $m$ is small. For $m$ large

$$
D_{m m} \sim\left(\sigma A^{2}\right)^{\frac{1}{2}} S /\left(\alpha S\left(\sigma A^{2}\right)^{\frac{1}{2}}+\Lambda E_{m}\right) .
$$

In both these expressions $\alpha$ is a complex constant of order unity.

This completes the construction of an estimate of $\Psi^{T}$. The equations (5.7) hold for $0<E<E_{m}<A^{2} \ll 1$ and $\sigma=\epsilon^{(1)} \sigma^{(1)} \sim A$. There is no restriction regarding the magnitudes of $m$ or $S$.

It is now possible to estimate $\epsilon^{(1)}$ by estimating $\mathbf{v}^{(2)} \cdot \hat{\mathbf{n}}$ and the integral (5.1). After considerable manipulation one obtains the leading order estimate

$$
\epsilon^{(1)} \sigma^{(1)} \sim\left(E A^{2} / E_{m}\right)^{\frac{1}{2}}+\gamma_{1}\left(A^{2} / \epsilon^{(1)}\right)+\gamma_{2}\left(A^{2} / \epsilon^{(1)}\right) D_{m m}
$$

where $\gamma_{1}$ and $\gamma_{2}$ are complex constants of order unity. Substituting for $D_{m m}$ from $(5.7 a)$ gives

$$
\sigma^{(1)}\left[\epsilon^{(1)}\right]^{2}-\left[\left(E A^{2} / E_{m}\right)^{\frac{1}{2}}+\gamma_{2}\left(S / N_{m}\right) A^{2} /\left(\alpha S\left(\sigma A^{2}\right)^{\frac{1}{2}}+\Lambda E_{m}\right)\right] \epsilon^{(1)}-\gamma_{1} A^{2}=0 .
$$


Because of the $\sigma^{\frac{1}{2}}$ term this is not a simple quadratic in $\epsilon^{(1)}$. By consideration of different values of $S$ one can construct table 4, which lists $\epsilon^{(1)}$ and the 'spin-up' time, equal to $\left[\omega \epsilon^{(1)}\right]^{-1}$. Recall once again the restrictions used in the calculation: $0<E<E_{m}<A^{2} \ll 1$.

\begin{tabular}{cccl}
$\begin{array}{c}\text { Parameter } \\
\text { range } \\
(1)\end{array}$ & \multicolumn{1}{c}{$S$} & \multicolumn{1}{c}{$\epsilon^{(1)}$} & $\tau=\left[\omega \epsilon^{(1)}\right]^{-1}$ \\
$(2)$ & $0 \leqslant S \ll 2 \Lambda E_{m} / A$ & $\pm A$ & $L / V_{A}$ \\
$(3)$ & $\Lambda E_{m} / A \epsilon^{\frac{1}{2}} \ll S$ & $\left\{\begin{array}{l}S A^{2} / \Lambda E_{m} \\
\Lambda E_{m} / S\end{array}\right\}$ & $\Lambda /\left(V_{A}^{2} 4 \pi \eta^{\prime}\right)$ \\
& $\left\{\begin{array}{l}A^{2} \\
A^{2}\end{array}\right\}$ & $\left(\omega L / V_{A}\right)^{\frac{2}{3} \omega^{-1}}$
\end{tabular}

TABLE 4. Characteristic scales for the pseudo-geostrophic mode

\section{Discussion}

In this paper three classes of waves have been mentioned and a detailed discussion of hydromagnetic boundary layers in a sphere and a thorough discussion of the pseudo-geostrophic modes have been given. From the last mentioned exercise it has been possible to estimate the spin-up time in the presence of a non-axisymmetric field.

The suggestion that the geomagnetic secular variation may be an expression of hydromagnetic waves has been made (e.g. Hide 1966; Braginskiy 1967; Malkus 1967). It is of interest to discuss this point in the context of this analysis. The first step is to calculate $E, E_{m}, A^{2}$ and $S$ for which the values of $\nu, \eta$ and $\eta^{\prime}$ are required. The core transport properties $\nu$ and $\eta$ are highly uncertain. A discussion of viscosity in the core is out of place here; such a discussion is currently in preparation. What is important here is that the magnetic Prandtl number $P r_{m}=E / E_{m}=4 \pi \eta v$ be small. The author has calculated $P r_{m}$ for thirteen metals (Al, $\mathrm{Sb}, \mathrm{Bi}, \mathrm{Cd}, \mathrm{Cs}, \mathrm{Ga}, \mathrm{Pb}, \mathrm{Hg}, \mathrm{K}, \mathrm{Ag}, \mathrm{Na}, \mathrm{Sn}, \mathrm{Zn}$ ) at temperatures in the vicinity of the melting point, using data from Smithells (1955), and obtained a range of values between $1.6 \times 10^{-7}(\mathrm{Bi})$ and $6.7 \times 10^{-6}(\mathrm{Al})$. Thus $\operatorname{Pr}_{m} \ll 1$ seems a reasonable assumption. The canonical value of $\eta$ is $3 \times 10^{-6} \mathrm{sec}^{-1}$ (Bullard 1949). Recently Stacey (1967) has reconsidered this number, allowing for alloying in the core, and has concluded that $\eta=3 \times 10^{-7} \mathrm{sec}^{-1}$ is more likely to be correct. This smaller value is accepted here. The other dimensional quantities are: $L=3.47 \times 10^{8} \mathrm{~cm}, \rho=10 \mathrm{~g} \mathrm{~cm}^{-3}, \omega=7.29 \times 10^{-5} \mathrm{sec}^{-1}, \eta^{\prime}=3 \times 10^{-9} \mathrm{sec}^{-1}$. The value of $\eta^{\prime}$ is from Eckhardt, Larner \& Madden (1963). It may be smaller. The calculation of conductivity profiles in the earth is not a simple task and cannot lead to a unique answer.

The choice of $B$ is not immediately obvious. The poloidal field at the coremantle boundary is of the order of $5 \mathrm{G}$. It is usually accepted that it remains of this order in the core but that there is a toroidal field of the order of $300 \mathrm{G}$ internally. In the discussion of the boundary layers it was made clear that the normal component of the magnetic field was important if the toroidal field did not exceed it by more than a factor of the inverse boundary-layer thickness. 
For even the fattest boundary layers earth-like parameters give $|\delta| \lesssim 10^{-4}$, so that the $300 \mathrm{G}$ toroidal field will not influence the boundary layers. Thus the decay rates of the various modes must be determined with $B=B_{r}=5 \mathrm{G}$.

For cases I and III, for which a truly non-dissipative expansion is relevant, it is perhaps more appropriate to take $B$ to be the toroidal field, of order $300 \mathrm{G}$. For case III it is to be remembered that the eigenvalue problem will involve boundary conditions other than the usual normal boundary conditions, and so indirectly involve the boundary layers.

For case II the dimensionless numbers are: $E \sim 10^{-15}, E_{m} \sim 3 \times 10^{-9}$, $A^{2} \sim 3 \times 10^{-10}, S \sim 10^{-3}$. For case I and III $A^{2}$ is replaced by $A_{T}^{2} \sim 10^{-6}$. Since $A_{T}^{2}$ is thirty times $E_{m}$ the dissipation-free analysis ought to be relevant and the MAC waves will have periods of the order of $10^{6}$ days, or 300 years, which are, in fact, not far from the characteristic time scales of the secular variation. A desirable and non-trivial exercise would be the solution of a MAC wave problem with the complete boundary conditions. Mantle conductivity is most probably important; its importance will be demonstrated for the pseudo-geostrophic modes. The relevant boundary conditions are wholly magnetic, as $A^{2} E / E_{m} \ll 1$.

The toroidal field may be estimated as follows. $S / E_{m} \sim 3 \times 10^{5}$, so that $\left[\epsilon^{(1)} S \mid E_{m}\right]^{\frac{1}{2}} \sim 1$, making $\Lambda \sim 1 . S A \sim 2 \times 10^{-8}$ so that the second term in the denominator of (5.7) will be dominant and $\Psi^{\top T} \sim S / E_{m} \epsilon^{(1)}$. This result applied to (5.8) leads to a quadratic for $\epsilon^{(1)}$, viz.

$$
\epsilon^{(1)^{2}}-\left[a\left(E A^{2} / E_{m}\right)^{\frac{1}{2}}+c A^{2} S / E_{m}\right] \epsilon^{(\mathbf{1})}-b A^{2}=0,
$$

where $a, b$ and $c$ are complex constants of order unity. Substituting the numerical values leads to

which gives

$$
\epsilon^{(1)^{2}}-\left[a \times 10^{-8}+c \times 10^{-4}\right] e^{(1)}-b \times 3 \times 10^{-10}=0,
$$

$$
\epsilon^{(1)} \approx\left\{\begin{array}{l}
c \times 10^{-4}, \\
-(b / c) \times 10^{-6} .
\end{array}\right.
$$

The quantity $1 / \epsilon^{(\mathbf{1})}$ represents the period of the disturbance in days. From (6.3) the geostrophic modes will have periods of the order of years to tens of years. Thus class II and class III waves may both contribute to the geomagnetic secular variation in different parts of the spectrum.

Finally, the question of spin-up can be briefly considered. Loper \& Benton (1970) have discussed spin-up between insulating flat plates in the presence of a uniform field for parameters such that $E / E_{m} \ll 1$ and $A^{2} / E_{m} \lesssim 1$. In their work $S=0$. They obtained a spin-up time of $\left(E_{m} / E A^{2}\right)^{\frac{1}{2}}$ for $A^{2} / E_{m}>1$ which is less than, or of the order of, the viscous spin-up time $E^{-\frac{1}{2}}$. The present work is not directly comparable because the constraining field here is not axisymmetric. This difference is more important than the different regions of parameter space.

Figure 1 shows a schematic diagram of a three parameter space defined by $E_{m}, A^{2}$ and $S$. Loper \& Benton's work applies to the left-hand half of the lower boundary. This work concerns the right-hand half of the diagram.

Since this paper reached its present form I have come across two further related papers neither of which duplicates the work reported above. On the 
question of hydromagnetic waves and the secular variation a second paper by Braginskiy (1970) has appeared and Loper (1971) has extended his spin-up work to the case of a conducting boundary.

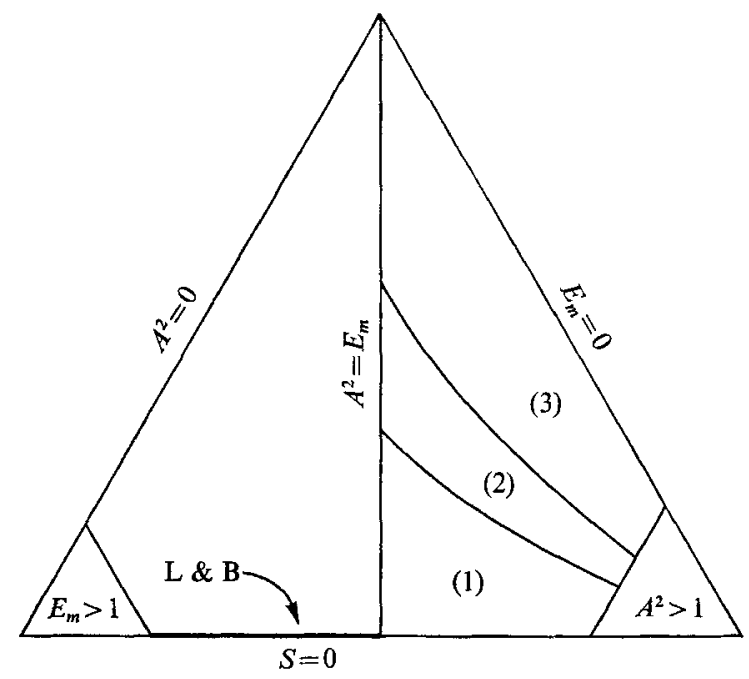

FIGURE 1. Schematic diagram of the three parameter space: $A^{2}, E_{m}, S$, numbers correspond to those in table $4 . \mathrm{L} \& \mathrm{~B}$ is the Loper \& Benton (1970) régime.

The comments of the referees regarding an earlier version of this paper are deeply appreciated. Support under the National Aeronautics and Space Administration Grant NGL-05-002-003 is gratefully acknowledged. This is contribution number 1975 of the Division of Geological and Planetary Sciences, California Institute of Technology.

\section{REFERENCES}

Benton, E. R. \& Loper, D. E. 1969 J. Fluid Mech. 39, 561-586.

Braginskiy, S. I. 1967 Geomag. \&e Aeron. 7, 851-859.

Braginskiy, S. I. 1970 Geomag. \& Aeron. 10, 172-181.

Bollard, E. C. 1949 Proc. Roy. Soc. A 197, 433-453.

ECKHARdt, D., Larner, K. \& Maddin, T. 1963 J. Geophys. Res. 68, 6279-6286.

Gans, R. F. $1971 J$. Fluid Mech. 45, 111-130.

Gremespan, H. P. 1968 The Theory of Rotating Fluids. Cambridge University Press.

HtDe, R. 1966 Phil. Trans. Roy. Soc. A 259, 615-647.

LOPER, D. E. 1971 Phys. Earth Planet. Interiors, 4, 129-137.

LOPER, D. E. \& BenTon, E. R. 1970 J. Fluid Mech. 43, 785-799.

Malkds, W. V. R. 1967 J. Fluid Mech. 28, 793-802.

Munk, W. H. \& MacDonald, G. J. F. 1960 The Rotation of the Earth. Cambridge University Press.

RoBERTs, P. H. 1967 An Introduction to Magnetohydrodynamics. New York: American Elsevier.

SnтtнeLLs, C. J. 1955 Metals Reference Book. Interscience.

SxAOY, F. D. 1967 Earth Planet. Sci. Letters, 3, 204-206. 